\title{
Comparison of Gradient Estimation in Cooperative Multi-Agent Source Seeking
}

\author{
Mochammad Sahal, Zulkifli Hidayat, Abdullah Alkaff \\ Dept. of Electrical Engineering \\ Sepuluh Nopember Institute of Technology \\ Surabaya, Indonesia \\ sahal@ee.its.ac.id, zulkifli@ee.its.ac.id, alkaff@ee.its.ac.id
}

\begin{abstract}
Agent-based source seeking problem is addressed in this paper. This problem is relevant in, e.g., in hazardous gas leak in a chemical disaster. In the cooperative search, agents develop a formation to effectively search the source by communicating one to another via a communication topology. The source, or the search target, is represented by a scalar field psi which might describe a temperature level, hazardous concentration of substances or vapor. Every agent has the information on its own position and the value of psi at any instance. The agents are identical modeled as single and double integrator. Consensus filter is used to control the agent formation and comparison three types of gradient estimation are employed to search the source. Experiments show that the proposed schemes give good performance to solve cooperative search for source seeking problem.
\end{abstract}

Keywords: cooperative source seeking, source seeking, formation control, gradient approximation.

\section{INTRODUCTION}

The problem of cooperative search for source seeking is still an interesting problem. There are publications in the literature on that problem that contribute to different cooperative search methods, different schemes, practical implementations with different kind of plants, e.g., unmanned aerial vehicles or mobile robots, to name some of common applications [1]

Source seeking algorithm is designed to drive single or multi agents to a source, where the source is represented by the scalar field signal $\psi$ and can be measured by all of agents. As the gradient of signal $\psi$ is measured, gradient climbing algorithm can be developed [2]. In this case, gradient approximation can be used from the distributed measurement of $\psi$ from the agents.

The first case, all measurements are performed by single agent [5] [6] as the position change in every instance. In this case, approximation can be done at least from two measurements from two different positions. Depending on the sensitivity of the sensor, the agent might need to move sufficiently far to obtain the gradient to converge to the source, but possibly with relatively large delay. In case no gradient can be computed, the agent will be stuck. The second case, multi agents cooperate [7] [8] to measure values of $\psi$ from different position simultaneously [3]. In this case, the search should be better with less possibility of stuck because of the multiple measurements of $\psi$ are available at every instant of gradient computation.

In this paper, we focus on comparison of gradient estimation on the source seeking from scalar field $\psi$ by using distributed multi-agent with a specified formation. There are $\mathrm{N}$ identical agents that are able to communicate in two directions. We show that the gradient of the scalar field $\psi$ can be approximated from the movement of the agents and directs the formation to the source. It is assumed that all agents are identical and every of them has sensors to measure the intensity of $\psi$ and its own position and relative to other agents. Each agent calculate the gradient based on its own measurement and those from its neighbors.

The remainder of this paper is arranged as follows. In Section II the required background is presented consisting of notation and problem description. In Section III the derivation of gradient approximation is described. Three gradient approximation methods, based on the leastsquares and the directional derivative are given. Analysis of consensus filter is given in Section IV followed by simulation experiments in Section V. Conclusions from the simulations are given in Section VI.

\section{BACKGROUND}

\section{A. Notation}

$\mathrm{G}=(\mathrm{V}, \mathrm{E})$ is a directed graph to model the interaction of agents, where the set of vertices $\mathrm{V}=\{1,2, \ldots, \mathrm{N}\}$ and set of edges $\mathrm{E} \subseteq \mathrm{V} \times \mathrm{V}$. Each vertex represents an agent and each edge is the communication link between two adjacent agents. . Edge $(i, j) \in E$ indicates that agent $i$ sends information to agent j. $\mathrm{N}_{\mathrm{i}}=\left\{\mathrm{i} \in \mathrm{V}: \mathrm{a}_{\mathrm{ij}} \neq 0\right\}$ is the set of neighbors of agent $i$.

Adjacency matrix $A=\left[a_{i j}\right] \in \mathbb{R}^{N \times N}$ for $a_{i j}=1$, if agent $i$ and agent $\mathrm{j} \in \mathrm{V}$ communicate each other and $\mathrm{a}_{\mathrm{ij}}=0$ otherwise. Note that $\mathrm{A}$ is symmetric.

Laplacian matrix $\mathrm{L}=\Delta-\mathrm{A}$, where $\Delta=\operatorname{diag}(\mathrm{A} \cdot \mathbf{1})$ is a diagonal matrix with the degree of an agents is the diagonal element, namely $\Delta_{\mathrm{ii}}=\mathrm{d}_{\mathrm{i}}=\Sigma_{\mathrm{j}} \mathrm{a}_{\mathrm{ij}}$ and $\mathbf{1}=\left[\begin{array}{lllll}1 & 1 & 1 & \ldots & 1\end{array}\right]^{\mathrm{T}} \in \mathbb{R}^{\mathrm{N}}$ is the vector with 1 as its elements. Right eigenvector $L$ is the eigenvector from the eigenvalue $\lambda_{1}=0$ and $L \cdot \mathbf{1}=0$. The second smallest eigenvalue $\lambda_{2}$ determines the convergence rate of the algorithm. $\left|\mathrm{N}_{\mathrm{i}}\right|$ is the number of neighbors in heighbor set $\mathrm{N}_{\mathrm{i}}$, dan $\otimes$ is the Kronecker product. 


\section{B. Problem Description}

Given a scalar field $\psi=\psi(\mathrm{r})$ which is a mapping $\psi: \mathbb{R}^{\mathrm{P}}$ $\rightarrow \mathbb{R}^{+}$, where $\mathrm{p}=1,2$, or 3 and $\mathrm{r} \in \mathbb{R}^{\mathrm{P}}$ that defines the position coordinate of the agent in space. For instance, $p=$ $3, r=\left[\begin{array}{ll}x & y\end{array}\right]^{T} \in \mathbb{R}^{P}$. The source is found indicated by the maximum value of $\psi$. Source seeking problem is to find the value of $r$ such that the scalar field $\psi$ is maximum. This can be formulated by the following optimization equation:

$$
\mathrm{r}^{*}=\arg \max \psi(\mathrm{r})
$$

In this paper, it is assumed that $\psi(r)$ has only a single maximum and has no local maximum as well.

\section{GRADIENT APPROXIMATION}

In the multi-agent based gradient approximation, each agent approximates its own gradient and the connected agents receive the approximations from its neighbors to compute the global gradient. We proposed three methods to compute the gradient approximation that based on the least squares.

\section{A. Approximation 1}

Each agent $\mathrm{i}$ measures the intensity of $\psi$ from its position. This is denoted by $\psi_{i}=\psi\left(r_{i}\right)$, where $i=1,2,3, \ldots$ $\mathrm{N}$. Given the position of agent $\mathrm{j}, \mathrm{r}_{\mathrm{j}}$, that is close to agent $\mathrm{i}$, $\mathrm{r}_{\mathrm{i}}$, the approximation can be calculated by using the Taylor series. The approximation of $\psi_{j}$ at $r_{i}$ is given by:

$$
\psi\left(\mathrm{r}_{\mathrm{j}}\right) \approx \psi\left(\mathrm{r}_{\mathrm{i}}\right)+\left(\mathrm{r}_{\mathrm{j}}-\mathrm{r}_{\mathrm{i}}\right)^{\mathrm{T}} \widehat{\boldsymbol{g}}\left(\mathrm{r}_{\mathrm{i}}\right)
$$

Agent $\mathrm{j}$ is neighboring agent that sends information to agent $\mathrm{i}, \widehat{\boldsymbol{g}}\left(\mathrm{r}_{\mathrm{i}}\right)$ is estimated gradient calculated by agent $\mathrm{i}$. For instance, $\mathrm{p}=3, \widehat{\boldsymbol{g}}\left(\mathrm{r}_{\mathrm{i}}\right)=\left[\widehat{\boldsymbol{g}}_{\boldsymbol{x}}\left(\mathrm{r}_{\mathrm{i}}\right) \widehat{\boldsymbol{g}}_{\boldsymbol{y}}\left(\mathrm{r}_{\mathrm{i}}\right) \widehat{\boldsymbol{g}}_{\boldsymbol{z}}\left(\mathrm{r}_{\mathrm{i}}\right)\right]^{\mathrm{T}}$. If agent $\mathrm{i}$ has $\left|\mathrm{N}_{\mathrm{i}}\right|$ neighbors, (1) becomes

$$
\begin{aligned}
{\left[\begin{array}{c}
\psi\left(r_{1}\right)-\psi\left(r_{i}\right) \\
\psi\left(r_{2}\right)-\psi\left(r_{i}\right) \\
\vdots \\
\psi\left(r_{\left|N_{i}\right|}\right)-\psi\left(r_{i}\right)
\end{array}\right] } & =\left[\begin{array}{c}
\left(r_{1}-r_{i}\right)^{T} \\
\left(r_{2}-r_{i}\right)^{T} \\
\vdots \\
\left(r_{\left|N_{i}\right|}-r_{i}\right)^{T}
\end{array}\right] \widehat{\boldsymbol{g}}\left(r_{i}\right) \\
b_{i} & =A_{i} \hat{g}_{i}
\end{aligned}
$$

where $b_{i} \in \mathbb{R}^{|\mathrm{Ni}| \times 1}, \mathrm{~A}_{\mathrm{i}} \in \mathbb{R}^{|\mathrm{Ni}| \times \mathrm{p}}$, dan $\widehat{\boldsymbol{g}}_{\boldsymbol{i}} \in \mathbb{R}^{\mathrm{p} \times 1}$. This problem can be solved by using the least squares method:

$$
\hat{g}_{i}=\left(A_{i}^{T} A_{i}\right)^{-1} A_{i}^{T} b_{i}
$$

\section{B. Approximation 2}

Normalization of (1) with the relative distance between agents and multiply it with the directed unit vector between agent $i$ and $j$ to become:

$$
\frac{\psi\left(r_{j}\right)-\psi\left(r_{i}\right)}{\left\|r_{j}-r_{i}\right\|} \frac{r_{j}-r_{i}}{\left\|r_{j}-r_{i}\right\|}=\frac{\left(r_{j}-r_{i}\right)^{T} \widehat{g}\left(r_{i}\right)}{\left\|r_{j}-r_{i}\right\|} \frac{r_{j}-r_{i}}{\left\|r_{j}-r_{i}\right\|}
$$

Equation (4) can be transformed into:

$$
\frac{\psi\left(r_{j}\right)-\psi\left(r_{i}\right)}{\left\|r_{j}-r_{i}\right\|^{2}}\left(r_{j}-r_{i}\right)=\frac{\left(r_{j}-r_{i}\right)^{T} \otimes\left(r_{j}-r_{i}\right)}{\left\|r_{j}-r_{i}\right\|^{2}} \widehat{\boldsymbol{g}}_{i}
$$

where $\mathrm{w}_{\mathrm{ji}}$ is the weighting factor between agent $\mathrm{i}$ and agent $\mathrm{j}$, defined by $\mathrm{w}_{\mathrm{ji}}=1 /\left\|\mathrm{r}_{\mathrm{j}}-\mathrm{r}_{\mathrm{i}}\right\|^{2}$. Equation (5) can be manipulated to add a weighting factor to each agent to form a weighting matrix $\mathrm{Wi}=\operatorname{diag}\left\{\mathrm{w}_{\mathrm{ji}}\right\}$. This is a $|\mathrm{Ni}| \times|\mathrm{Ni}|$ diagonal matrix containing weighting factors to all $|\mathrm{Ni}|$ agents that send information to agent i. The related matrices are written as:

$W_{i}=\left[\begin{array}{ccc}\frac{1}{\left\|r_{1}-r_{i}\right\|^{2}} & \cdots & 0 \\ \vdots & \ddots & \vdots \\ 0 & \cdots & \frac{1}{\left\|r_{\left|N_{i}\right|}-r_{i}\right\|^{2}}\end{array}\right]$

$b_{i}=\left[\begin{array}{c}\left(\psi\left(r_{1}\right)-\psi\left(r_{i}\right)\right)\left(r_{1}-r_{i}\right) \\ \left(\psi\left(r_{2}\right)-\psi\left(r_{i}\right)\right)\left(r_{2}-r_{i}\right) \\ \vdots \\ \left(\psi\left(r_{\left|N_{i}\right|}\right)-\psi\left(r_{i}\right)\right)\left(r_{\left|N_{i}\right|}-r_{i}\right)\end{array}\right]$

$A_{i}=\left[\begin{array}{c}\left(r_{1}-r_{i}\right)^{T} \otimes\left(r_{1}-r_{i}\right) \\ \left(r_{2}-r_{i}\right)^{T} \otimes\left(r_{2}-r_{i}\right) \\ \vdots \\ \left(r_{\left|N_{i}\right|}-r_{i}\right)^{T} \otimes\left(r_{\left|N_{i}\right|}-r_{i}\right)\end{array}\right]$

and the least squares formulation can compactly written as:

$$
W_{i} A_{i} \hat{g}_{i}=W_{i} b_{i}
$$

By using the least squares:

$$
\hat{g}_{i}=\left(A_{i}^{T} W_{i}^{2} A_{i}\right)^{-1} A_{i}^{T} W_{i}^{2} b_{i}
$$

\section{Approximation 3}

This approximation can be computed as the average of the slope in the direction of normalization vector, expressed as:

$$
\hat{g}_{i}=\frac{1}{\left|N_{i}\right|} \sum_{j \in N_{i}} \frac{\psi\left(r_{j}\right)-\psi\left(r_{i}\right)}{\left\|r_{j}-r_{i}\right\|^{2}}\left(r_{j}-r_{i}\right)
$$

To compare these approximations, the Mean Squared Error (MSE) and the Root Mean Squared Error (RMSE) will be calculated and analyzed on the simulation section. The formulation of MSE is $\frac{1}{n} \sum_{i=1}^{n}\left(r_{o i}-r_{j i}\right)^{2}$ and RMSE is $\sqrt{\left(\frac{1}{n} \sum_{i=1}^{n}\left(x_{o i}-x_{j i}\right)^{2}\right)}$, where $\mathrm{r}$ is position $\left([\mathrm{x} \mathrm{y} \mathrm{z}]^{\mathrm{T}}\right), \mathrm{o}$ is the original gradient, $\mathrm{j}$-approximation, $\mathrm{i}$-datum, and $n$ number of data.

\section{GRADIENT OF THE CONSENSUS FILTER}

We propose a consensus algorithm that modifies one in [9]. 
$\dot{g}_{i}=\beta \sum_{j \epsilon N_{i}} a_{i j} e_{g_{i j}}(t)+\beta\left(1+d_{i}\right)\left(\hat{g}_{i}(t)-g_{i}(t)\right)$

where

$$
\begin{aligned}
& e_{g_{i j}}(t)=\left(\hat{g}_{i}(t)-g_{i}(t)\right)-\left(\hat{g}_{j}(t)-g_{j}(t)\right) \\
& e_{g_{i j}}(t)=\left(\hat{g}_{i}(t)-g_{j}(t)\right)-\left(\hat{g}_{i}(t)-g_{j}(t)\right)
\end{aligned}
$$

and $\beta \geq 1$ is a control parameter to tracking performance of the gradient as the agent moves. By using the definition of the Laplacian graph, the following equation is obtained:

$$
\begin{aligned}
\dot{g}= & -\beta\left(I_{N} \otimes I_{p}+\Delta \otimes I_{p}+L \otimes I_{p}\right) g \\
& +\beta\left(I_{N} \otimes I_{p}+\Delta \otimes I_{p}+L \otimes I_{p}\right) \hat{g}
\end{aligned}
$$

so that the previous equation becomes:

$$
\dot{g}=\beta(-A g+A \hat{g})
$$

\section{SiMULATION RESULTS}

For the simulation experiment, the following scalar field function is used:

$$
\psi(x, y)=50 e^{-\frac{(x-65)^{2}}{1800}-\frac{(y-70)^{2}}{11250}}+50 e^{-\frac{(x-65)^{2}}{16200}-\frac{(y-70)^{2}}{1250}}
$$

and the original gradient is obtained analytically from the gradient $\psi(x, y)$ :

$$
\nabla \psi(x, y)=\frac{\partial \psi}{\partial x} i+\frac{\partial \psi}{\partial y} j
$$

where

$$
\begin{gathered}
\frac{\partial \psi}{\partial x}=-\frac{x-65}{900} e^{-\frac{(x-65)^{2}}{1800}-\frac{(y-70)^{2}}{11250}} \\
\frac{\partial \psi}{\partial y}=-\frac{x-65}{5625} e^{-\frac{(x-65)^{2}}{1800}-\frac{(y-70)^{2}}{11250}} \\
-\frac{y-700}{1800}-\frac{(y-70)^{2}}{11250} \\
-\frac{y-70}{-\frac{(x-65)^{2}}{1800}-\frac{(y-70)^{2}}{11250}}
\end{gathered}
$$

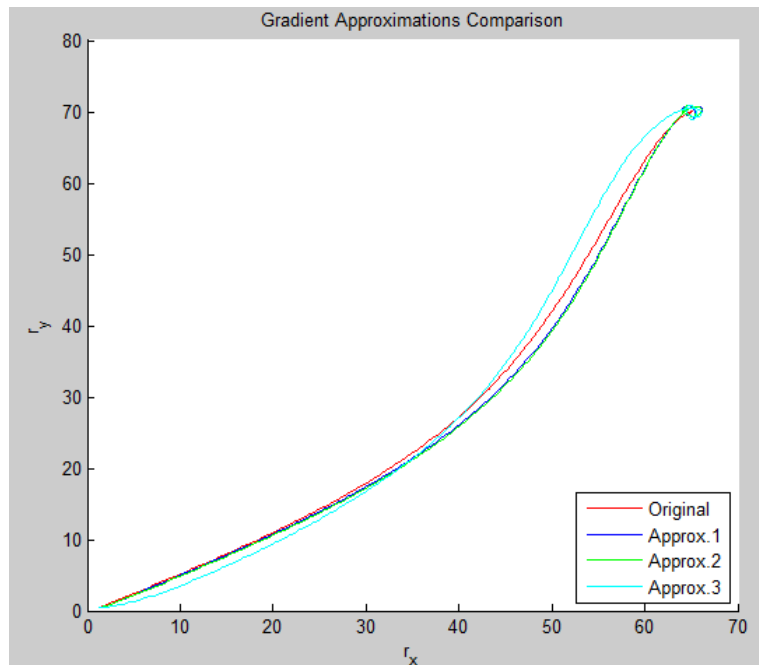

Fig. 1. $r_{x}-r_{y}$ gradient approximation result

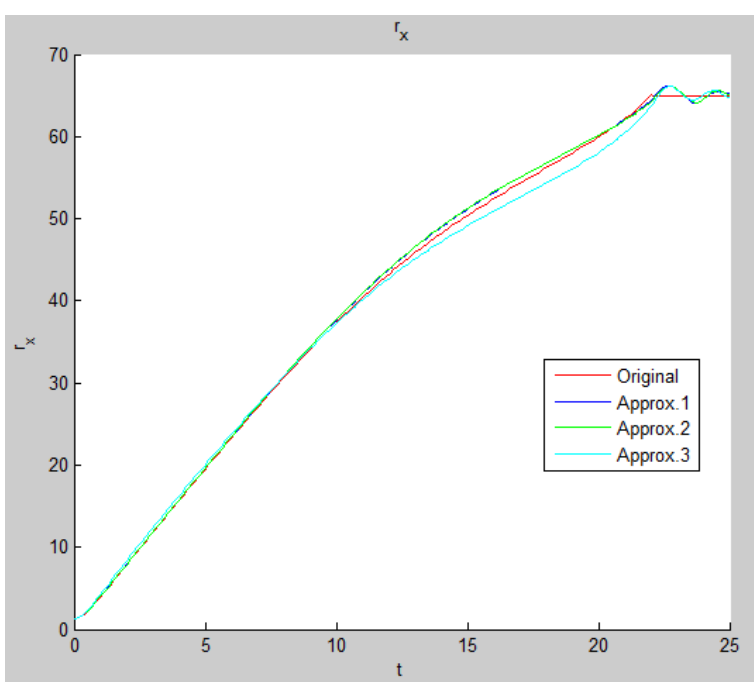

Fig. 2. $r_{x}$ gradient approximation result

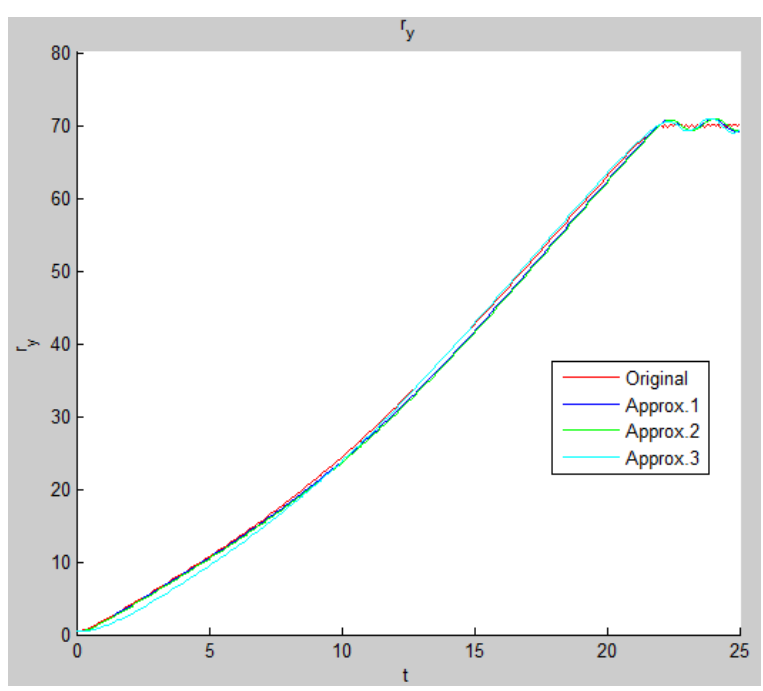

Fig. 3. $r_{y}$ gradient approximation result

Each agent is an identical agent with single integrator model.

TABLE 1. COMPARISONS OF MSE, RMSE VS APPROX

\begin{tabular}{|l|l|l|l|l|}
\hline Statistics & $\mathrm{r}_{\mathrm{i}}$ & Approx1 & Approx2 & Approx3 \\
\hline MSE & $\mathrm{r}_{\mathrm{x}}$ & 0.2253 & 0.2538 & 1.0332 \\
\hline & $\mathrm{r}_{\mathrm{y}}$ & 0.3375 & 0.5515 & 0.5556 \\
\hline & $\mathrm{r}_{\mathrm{T}}$ & 0.5628 & 0.8053 & 1.5888 \\
\hline RMSE & $\mathrm{r}_{\mathrm{x}}$ & 0.4747 & 0.5038 & 1.0164 \\
\hline & $\mathrm{r}_{\mathrm{y}}$ & 0.5810 & 0.7426 & 0.7454 \\
\hline & $\mathrm{r}_{\mathrm{T}}$ & 1.0567 & 1.2464 & 1.7618 \\
\hline
\end{tabular}




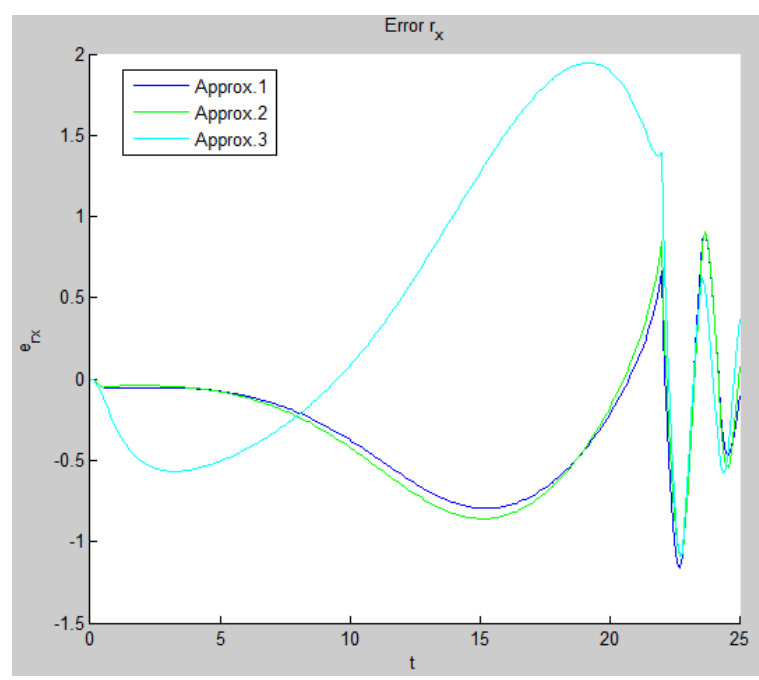

Fig. 4. Error $r_{x}$

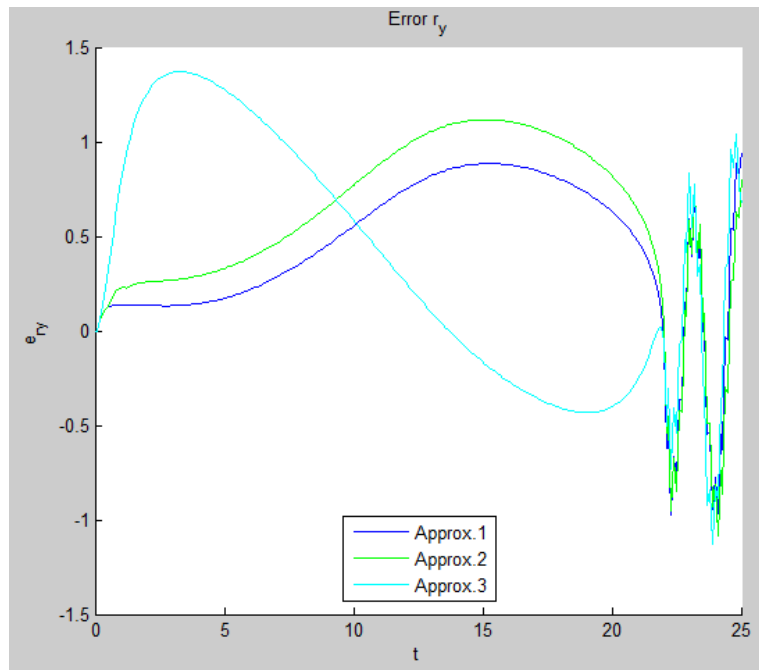

Fig. 5. Error $r_{y}$

The MSE and RMSE of the error of error $r_{x}, r_{y}$ dan $r_{T}$ with respect to the original gradient of each approximation method are shown in Table 1. From the table, it can be seen that Approximation 1 is better that the other approximation methods because of the smaller MSE and RMSE. However, Approximation 2 and Approximation 3 are also sufficiently good since Figure 1 to Figure 6 show the approximations is close enough to the original gradient.

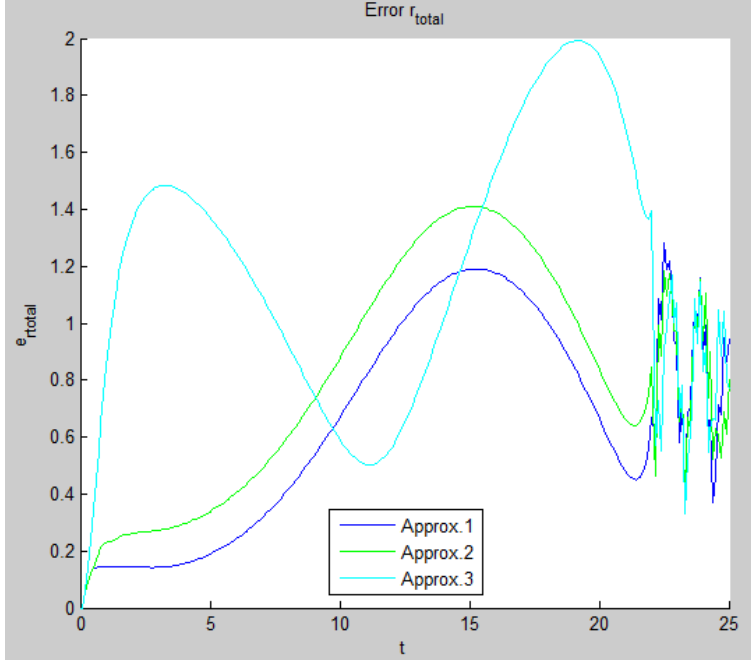

Fig. 6. Error $\mathrm{r}_{\text {total }}$

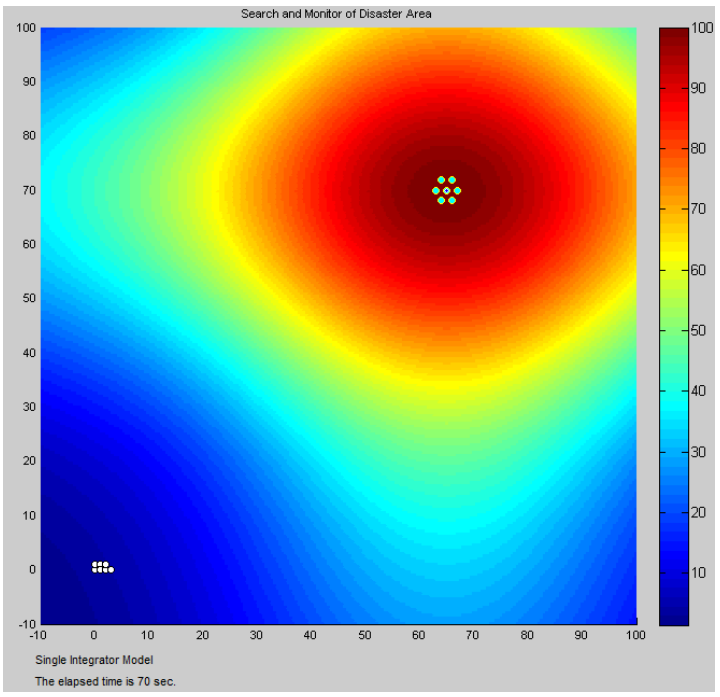

Fig. 7. Trajectory of the formation from initial condition to the source.

Figure 1 show the evolution of the heading of the agent from the initial value until the source is located. Figure 1 can be detailed by Figure 2 and Figure 3 with respect to time function of $r_{x}$ dan $r_{y}$.

Figure 4 and Figure 5 are respectively the error of $r_{x}$ and $r_{y}$ with respect to the original gradient. Figure 6 is the combination between Figure 4 and Figure 5.

Figure 7 and Figure 8 shows the trajectory of the cooperative multi-agent from the initial condition to the source by using all three gradient approximation methods. 


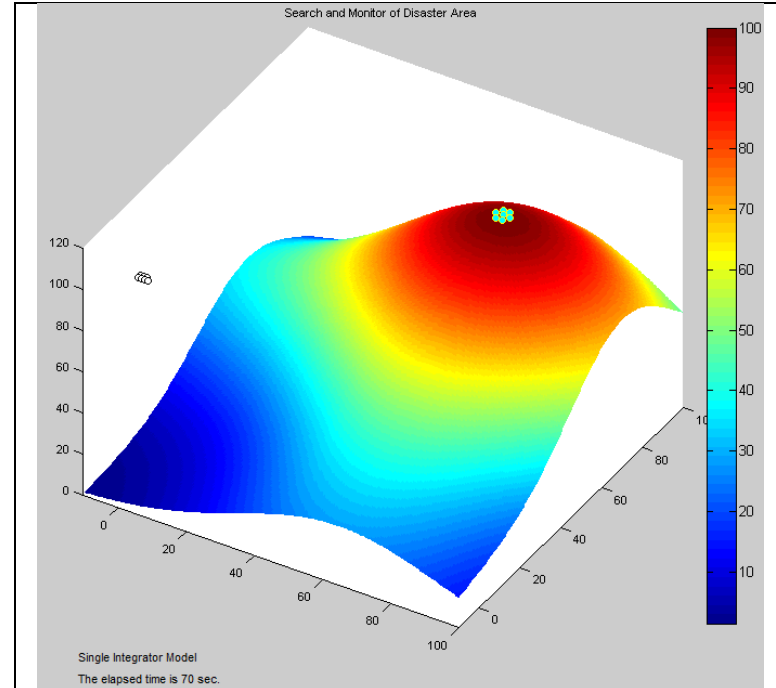

Fig. 8. Trajectory of the formation in 3D

\section{CONCLUSION}

Three gradient approximation methods have been presented in this paper. All methods are based on the solution of the least-squares method and applied to source seeking problem by a multi-agent system.

From the simulation it can be concluded that the experiment schemes by using the gradient estimation is interesting and has a promising for further development and applications. Compared to Approximation 2 and Approximation 3, Approximation 1 is better with respect to the values of MSE and RMSE. However, the other approximations give sufficiently results as well.

For further research, scalar field functions containing local maximums can be used to have a more complex problem. More complex schemes can also be developed to tackle more problems.

\section{REFERENCES}

[1] A. S. Matveev, H. Teimoori, and A. V. Savkin, Navigation of a unicycle-like mobile robot for environmental extremum seeking," Automatica, vol. 47, no. 1, pp. 85 - 91, 2011.

[2] R. Bachmayer and N. Leonard, "Vehicle networks for gradient descent in a sampled environment," in Decision and Control, 2002, Proceedings of the 41st IEEE Conference on, vol. 1, dec. 2002, pp. 112 - 117 vol.1

[3] P. Ogren, E. Fiorelli, and N. Leonard, "Cooperative control of mobile sensor networks:adaptive gradient climbing in a distributed environment," Automatic Control, IEEE Transactions on, vol. 49, no. 8, pp. 1292 - 1302, aug. 2004.

[4] R. Olfati-Saber and J. S. Shamma, "Consensus filters for sensor networks and distributed sensor fusion," in Decision and Control, 2005 and 2005 European Control Conference. CDC-ECC 05. 44th IEEE Conference on, dec. 2005, pp. 6698 - 6703..

[5] J. Cochran and M. Krstic. Nonholonomic source seeking with tuning of angular velocity. Automatic Control, IEEE Transactions on, 54(4):717 -731, april 2009.

[6] J. Cochran, A. Siranosian, N. Ghods, and M. Krstic. 3-d source seeking for underactuated vehicles without position measurement. Robotics, IEEE Transactions on, 25(1):117-129, feb. 2009.

[7] J. Cortes. Achieving coordination tasks in finite time via nonsmooth gradient flows. In Decision and Control, 2005 and 2005 European Control Conference. CDC-ECC '05. 44th IEEE Conference on, pages 6376-6381, dec. 2005.

[8] N. Ghods and M. Krstic. Multi-agent deployment around a source in one dimension by extremum seeking. In American Control Conference (ACC), 2010, pages 4794 -4799, 30 2010-july 2 2010.

[9] Esteban Rosero and Herbert Werner. Cooperative Source Seeking via Gradient Estimation and Formation Control. 2014 UKACC International Conference on Control 9th - 11th July 2014, Loughborough, U.K. 